\title{
Editorial
}

\section{From the Desk of the Editor}

\author{
Pradeep Singh ${ }^{1}$ Shraddha Singhania, ${ }^{2,0}$ Sohael M. Khan ${ }^{3}$ \\ ${ }^{1}$ Dr. L. H. Hiranandani Hospital, Powai, Mumbai, India \\ 2Department of Radiodiagnosis, AVBRH, Wardha, Maharashtra, \\ India \\ ${ }^{3}$ Department of Orthopaedics and Spine Services, AVBRH, Wardha, \\ India \\ Int J Recent Surg Med Sci 2019;5:35
}

Endovascular surgery was first described in 1904 and has continuously evolved ever since. Repeated work in the same area and more experience led to good clinical results. The elegance of the endovascular approach has limited the efficiency of embolization. Exploring new technologies has made endovascular techniques not only safer but also as effective as microsurgery.

As the Editors, we have great privilege of releasing this issue based on endovascular surgery. The issue is in collaboration with Department of Endovascular Surgery, MS Ramaiah Hospital, Bengaluru, under the guidance of Dr. Sanjay Desai, Head of the Department.

Vascular and endovascular surgery is rapidly advancing. Spectrum and complexity of vascular diseases in India is relatively unknown making our country a rich ground for researches and exploration. This issue, therefore, aims to be a showcase for the original work being done in endovascular surgery in our country. It also endeavors to reflect the important work of vascular surgeons contributing to vascular education, research, quality initiatives, and clinical practice in our country, to strive for excellence.

Being spine surgeons, we have always supported and understood that the vascular expertise is important in many spinal operative procedures as chances of minor venous injuries are always high during exposure and instrumentation. Hence, knowledge of vascular repair is a must for all spine surgeons and also vascular surgeon involvement helps in prompt repair of vascular surgeries. Hence, with this issue we wish to bring focus on a very important branch of surgery and developments that have taken place since vascular surgery was first introduced.

Concluding with confession of a surgeon:

Life behind OR door...is just three words... Good, bad, or complicated...!

\section{Conflict of Interest}

None declared.
Address for correspondence

Shraddha Singhania, DMRD,

Department of Radiodiagnosis, AVBRH,

Wardha, Maharashtra 442001, India

(e-mail: shraddha2527@gmail.com).
DOI https://doi.org/

10.1055/s-0039-3402324

ISSN 2455-7420.
(C)2019 Medical and Surgical

Update Society
License terms

$($ (1) (1) $\Theta \circledast$ 